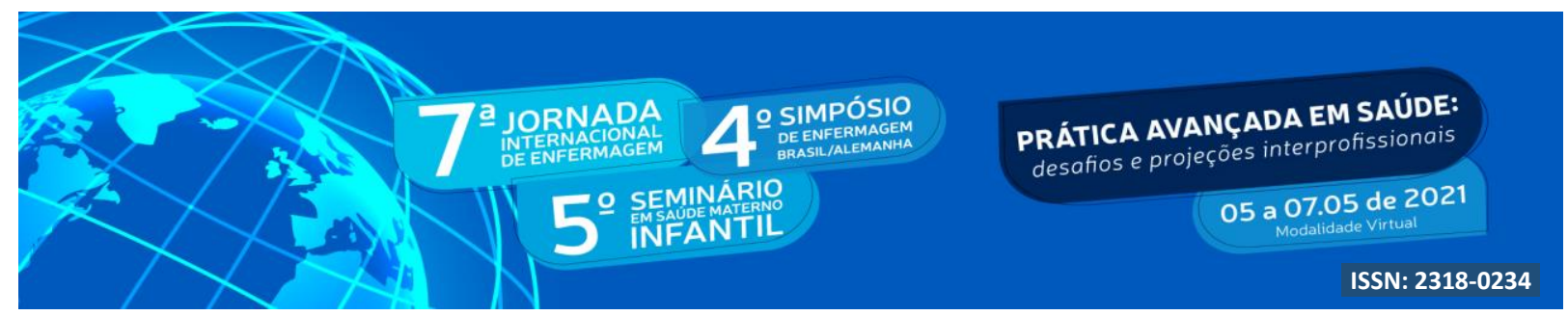

DOI: http://doi.org/10.48195/jie2021-078

\title{
COMPORTAMENTO AUTOLESIVO EM ADOLESCENTES: REVISÃO INTEGRATIVA DA LITERATURA ${ }^{1}$
}

\author{
Francielle Morais de Paula ${ }^{2}$; Francielle Brum dos Santos de Siqueira ${ }^{3}$; Camila Freitas \\ Hausen $^{4}$; Diúlia Calegari de Oliveira ${ }^{5}$;Graciela Dutra Sehnem ${ }^{6}$; Eliane Tatsch Neves ${ }^{7}$.

\section{RESUMO}

Objetivo: Identificar as evidências científicas acerca do comportamento autolesivo em adolescentes. Método: Trata-se de uma revisão integrativa da literatura. O levantamento bibliográfico foi realizado no mês de agosto de 2020 na base de dados eletrônica Literatura Latino-americana e do Caribe em Ciências da Saúde (LILACS). O corpus do estudo foi composto por 11 artigos. Resultados: Verificou-se que a prevalência das autolesões são justificadas pela necessidade de produzir alívio momentâneo, buscar autoafirmação e atenuar sensações negativas como tristeza. Aponta-se, na literatura, a alta incidência de adolescentes do sexo feminino, apresentando-se como as principais protagonizadoras deste fenômeno. Conclusões: Conclui-se que as evidências científicas relacionadas ao comportamento autolesivo entre adolescentes abordam principalmente as causas e fatores envolvidos no desenvolvimento dessa conduta.

Palavras-chave: Comportamento autolesivo; Adolescentes; Enfermagem.

\begin{abstract}
Objective: To identify the scientific evidence about self-injurious behavior in adolescents. Method: This is an integrative literature review. The bibliographic survey was carried out in August 2020 in the electronic database of Latin American and Caribbean Literature in Health Sciences (LILACS). The study corpus was composed of 11 articles. Results: It was found that the prevalence of self-harm is justified by the need to produce momentary relief, seek self-assertion and mitigate negative feelings such as sadness. In the literature, the high incidence of female adolescents is pointed out, presenting themselves as the main protagonists of this phenomenon. Conclusions: It is concluded that the scientific evidence related to self-injurious behavior among adolescents mainly addresses the causes and factors involved in the development of this conduct.
\end{abstract}

Key Words: Self-injurious behavior; Adolescents; Nursing.

\footnotetext{
${ }^{1}$ Estudo de revisão integrativa da literatura;

${ }^{2}$ Enf $^{a}$ Mestranda, Universidade Federal de Santa Maria; fraanmdepaula@gmail.com

3 Enf $^{a}$ Mestranda, Universidade Federal de Santa Maria; francisbrum@gmail.com

4 Enf $^{a}$ Mestranda, Universidade Federal de Santa Maria; camilafht_@ hotmail.com

5. Acadêmica do curso de Enfermagem, Universidade Federal de Santa Maria; diuliacoliveira@gmail.com

6.Coorientadora. Enf ${ }^{a}$ Dra, Universidade Federal de Santa Maria; graci_dutra@yahoo.com.br

7.Orientadora. Enf ${ }^{a}$ Dra, Universidade Federal de Santa Maria; eliane.neves@ufsm.br
} 


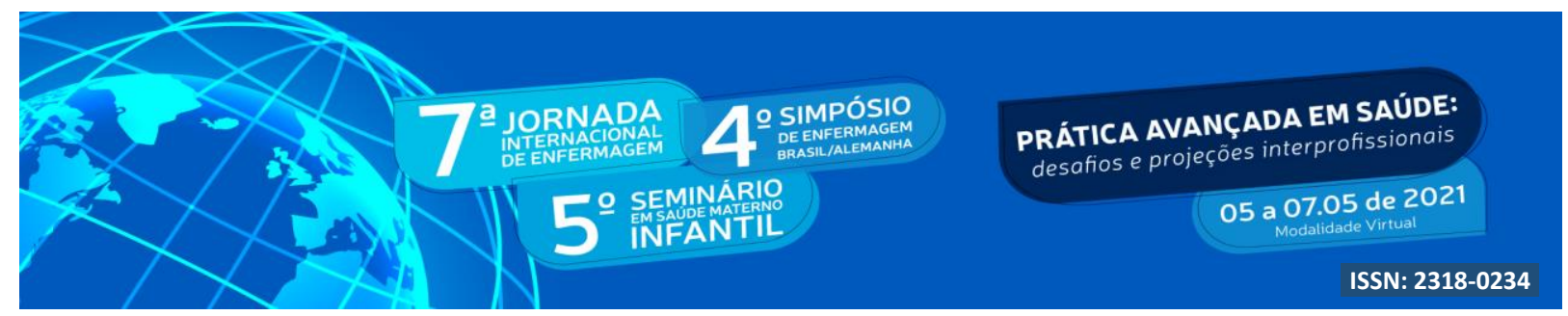

\section{INTRODUÇÃO}

O comportamento autolesivo se caracteriza por ser aquele no qual o indivíduo é capaz de produzir lesões contra si mesmo. Tal conduta pode surgir com ideação suicida ou não, recebendo conceitos diferentes e se manifestando por causas distintas (SILVA; SIQUEIRA, 2017). A autolesão sem intenção suicida pode ser compreendida por ser uma ação com propósito de produzir alívio, já a autolesão com intenção suicida, desencadea-se com o intuito de provocar a própria morte.

Ademais, suas formas de expressão, diferem-se sendo o comportamento autolesivo sem intenção suicida, geralmente manifestado na forma de arranhões, cortes superficiais e/ou queimaduras contra o próprio corpo (CUMMINGS, 2015). No que se refere às lesões com intenção suicida, a maioria ocorre na forma de intoxicação exógena. O suicídio consumado está entre as 10 principais causas de óbito no mundo, vitimando principalmente jovens e adultos. No entanto, tal conduta, apresenta-se de forma desigual por todo o mundo (WHO, 2014).

Estima-se, que o comportamento suicida possui fatores de risco associados, tais como problemas existenciais, biológicos, médicos e motivações sociais (WHO, 2014). Já o comportamento autolesivo sem intenção suicida possui mecanismo de enfretamento, onde o indivíduo busca por meio dele o alívio, mesmo que momentâneo, em decorrência de eventos estressores (SANTOS; FARO, 2018).

Condutas autolesivas ocorrem em diferentes ciclos de vida, recebendo destaque na adolescência e sendo predominante no sexo feminino (KLONSKY et al., 2015). Tal fato é decorrente de diversas causas, sobretudo, emocionais, sendo conduzida na forma de alívio perante sensações ruins como vazio e desprezo (FONSECA et al., 2018).Esta fase da vida é responsável por ser um período de transição entre a infância e a vida adulta. É neste período que ocorrem as principais transformações físicas, psíquicas e emocionais no ser humano (SANTOS; FARO, 2018).

Neste viés, por se tratar de um período, muitas vezes, conflituoso, o comportamento autolesivo pode ocorrer como "válvula de escape" diante de situações traumáticas que 


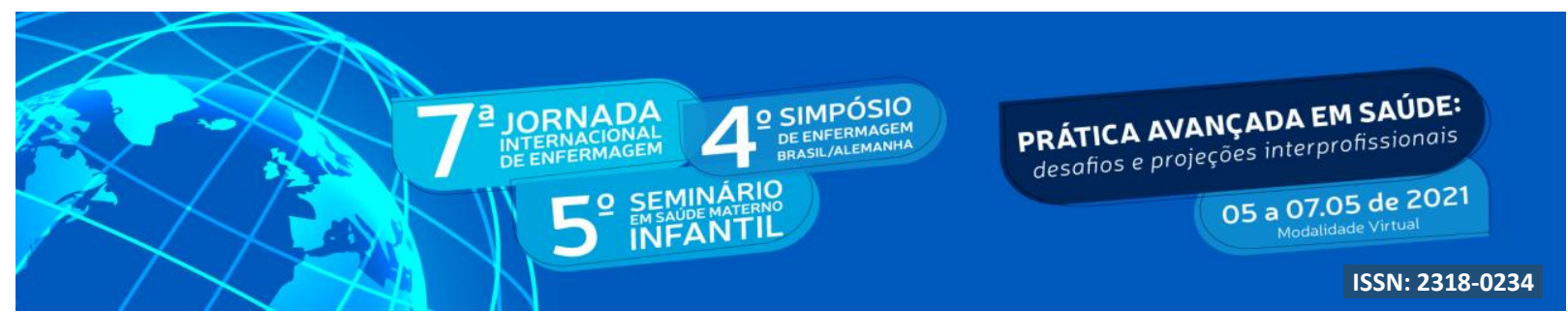

envolvem adolescentes e que podem ter emergido na infância. A busca por alívio de sentimentos negativos e a necessidade de compreender emoções são vistas como algumas causas para este comportamento (RAUPP; MAURIN; MOSMANN, 2018).

Para atuar nesse contexto, se faz necessário preparar profissionais de saúde para atenderem as demandas, cada vez maiores, de casos de jovens que aderem ao comportamento autolesivo. Dessa forma, torna-se mister a mobilização e envolvimento de instituições acadêmicas e órgãos governamentais para a capacitação dos mesmos

Dessa forma, este trabalho justifica-se pela necessidade de investigação sobre a temática do comportamento autolesivo entre adolescentes, visto a demanda crescente de adolescentes que desenvolvem esse comportamento (RAUPP; MAURIN; MOSMANN, 2018). Assim, com o entendimento de tal comportamento, sobretudo dos mecanismos envolvidos neste processo, torna-se possível pensar em estratégias de intervenção a fim de prevenir incidências. Diante das considerações acima relatas, este estudo tem como questão norteadora: Quais as evidências científicas relacionadas ao comportamento autolesivo em adolescentes?

\section{OBJETIVO}

Identificar as evidências científicas acerca do comportamento autolesivo em adolescentes.

\section{METODOLOGIA}

Trata-se de uma revisão integrativa da literatura que se conceitua como um tipo de estudo que permite através de evidências, a associação de saberes e inclusão de resultados de estudos na prática clínica (Sousa et al., 2017). Para a realização do estudo foram percorridas e desenvolvidas seis etapas: identificação do tema e formulação da questão de pesquisa; estabelecimento de critérios para inclusão e exclusão de estudos; definição das informações a serem extraídas dos estudos selecionados/categorização dos estudos; avaliação dos estudos incluídos na revisão integrativa; interpretação dos resultados e, por último, apresentação da 


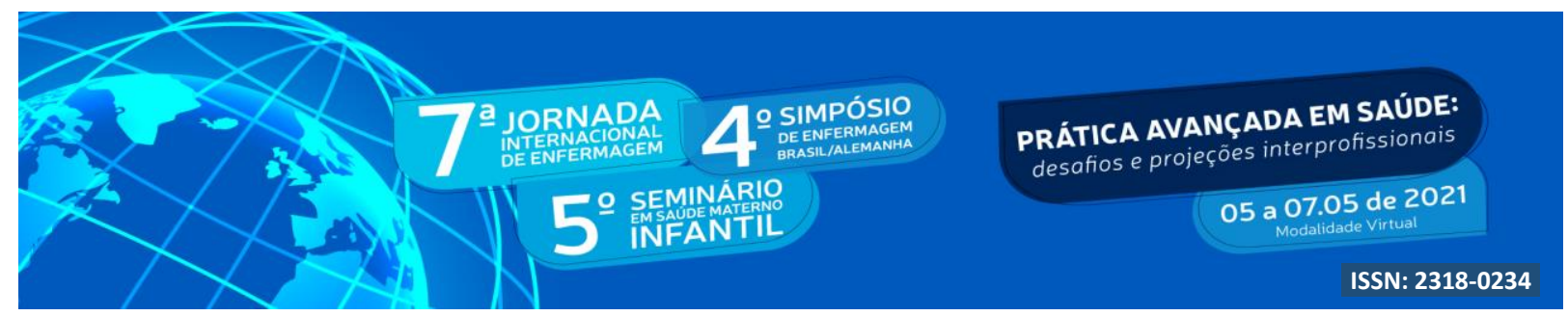

síntese do conhecimento (MENDES, SILVEIRA \& GALVÃO, 2008).

Os critérios de inclusão estabelecidos foram: artigos originais voltados para a temática e que atendessem a questão de pesquisa. Os critérios para exclusão foram os artigos com textos indisponíveis. O levantamento bibliográfico foi realizado no mês de agosto de 2020 nas bases de dados eletrônicas Literatura Latino-americana e do Caribe em Ciências da Saúde (LILACS). As bases de dados foram definidas por compreender que estas englobam a literatura publicada nos países da América Latina e Caribe, como também referências técnicocientíficas brasileiras, além de incluírem periódicos conceituados para a área da saúde.

Para tanto, foram utilizados, os seguintes descritores e operadores boleanos: [Adolescente] AND [Comportamento Autodestrutivo].Na busca, foram localizados 40 produções. Após leitura criteriosa e análise das mesmas, o estudo resultou em 11 artigos que constituiram o corpus do estudo. Assim, na figura 1, apresenta-se o fluxograma relacionado ao processo de seleção dos artigos. Por fim, no que se refere aos aspectos éticos, foram respeitadas as ideias, as definições e os conceitos empregados pelos autores dos estudos analisados, enfatizando que os mesmos foram citados de forma cabível e respeitável.

Figura 1. Fluxograma de processo de busca e seleção de artigos.

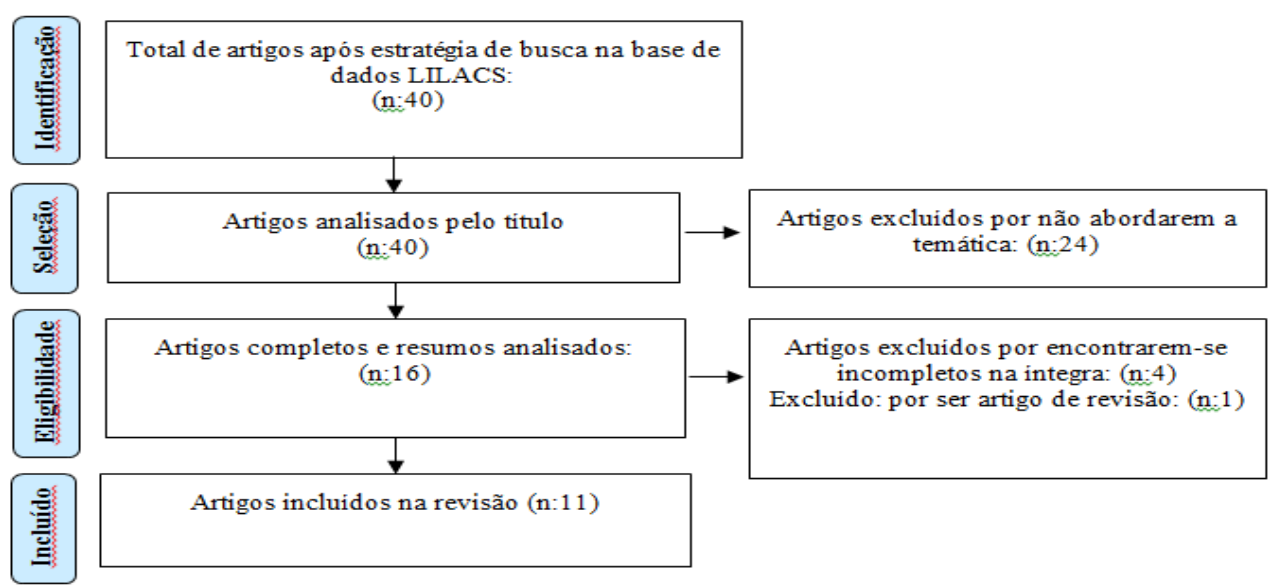

Fonte: Figura elaborada pela própria autora com base no diagrama PRISMA (MOHER et al., 2015). 


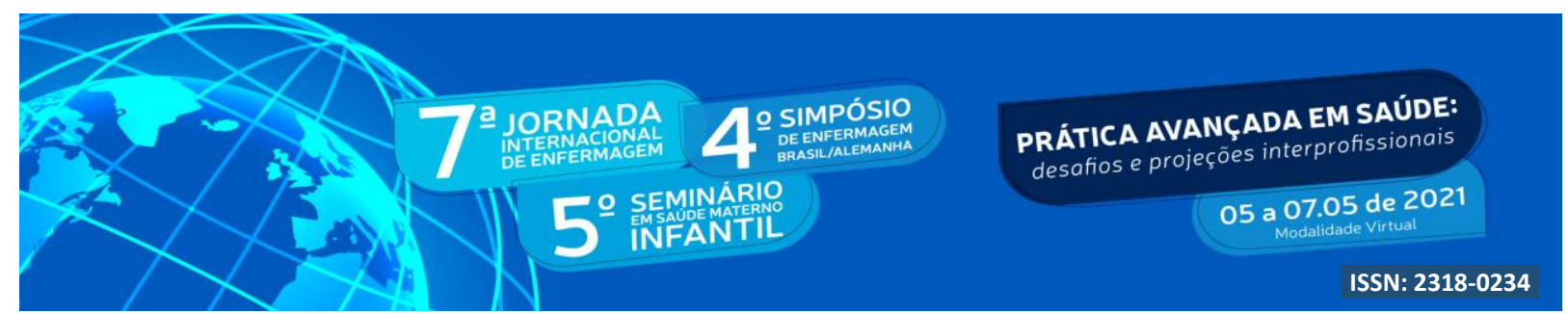

\section{RESULTADOS E DISCUSSÃO}

O corpus do estudo foi composto por 11 artigos. A seguir, o Quadro 1 sintetiza os artigos que compuseram a revisão.

Quadro 1: Síntese dos artigos selecionados para a revisão integrativa, segundo autores/ano, título do trabalho, delineamento metodológico e país de realização/periódico.

\begin{tabular}{|c|c|c|c|c|}
\hline ID & Autores/ano & Título & $\begin{array}{l}\text { Delineamento } \\
\text { metodológico }\end{array}$ & $\begin{array}{l}\text { País de } \\
\text { realização/ } \\
\text { Periódico }\end{array}$ \\
\hline 1. & $\begin{array}{l}\text { Carvalho, C. B. et al., } \\
\text { / } 2017\end{array}$ & $\begin{array}{l}\text { Biting myself so I don't } \\
\text { bite the dust: prevalence } \\
\text { and predictors of } \\
\text { deliberate self-harm and } \\
\text { suicide ideation in } \\
\text { Azorean youths }\end{array}$ & Misto & $\begin{array}{l}\text { Portugal / } \\
\text { Revista } \\
\text { Brasileira } \\
\text { Psiquiátrica }\end{array}$ \\
\hline 2. & $\begin{array}{l}\text { Ibáñeza, } \\
\text { Fernández, }\end{array}$ & $\begin{array}{l}\text { Adolescente con } \\
\text { autolesiones no suicidas } \\
\text { en un entorno de } \\
\text { adversidad psicossocial }\end{array}$ & $\begin{array}{l}\text { Estudo de } \\
\text { Caso }\end{array}$ & $\begin{array}{l}\text { Espanha / } \\
\text { Arch. argent. } \\
\text { Pediatr. }\end{array}$ \\
\hline 3. & Gonzalo et al., / 2018 & $\begin{array}{l}\text { Comportamientos } \\
\text { autolesivos } \\
\text { adolescentes: } \quad \text { Estudio } \\
\text { cualitativo } \\
\text { características, } \\
\text { significados y contextos }\end{array}$ & $\begin{array}{l}\text { Qualitativo } \\
\text { Transversal }\end{array}$ & $\begin{array}{l}\text { Argentina } \quad / \\
\text { Arch. argent. } \\
\text { Pediatr. }\end{array}$ \\
\hline
\end{tabular}




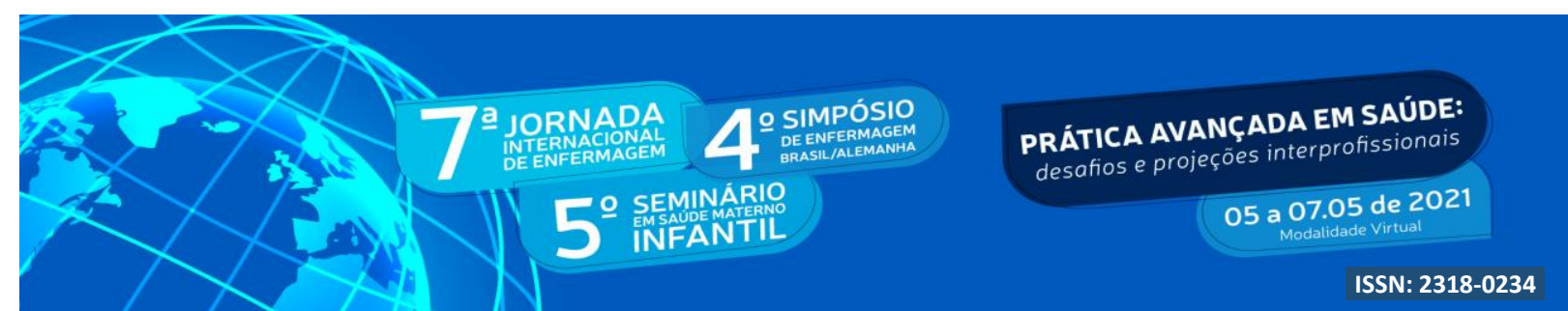

\begin{tabular}{|c|c|c|c|c|}
\hline 4. & Barbosa et al., / 2019 & $\begin{array}{l}\text { A prática de autolesão } \\
\text { em jovens: uma dor a ser } \\
\text { analisada }\end{array}$ & Qualitativo & $\begin{array}{l}\text { Brasil /Revista } \\
\text { Mineira de } \\
\text { Enfermagem }\end{array}$ \\
\hline 5. & Maio et al., / 2019 & $\begin{array}{l}\text { Autoagresiones en } \\
\text { adolescentes atendidos } \\
\text { en un hospital de alta } \\
\text { complejidad: Serie de } \\
\text { casos clínicos }\end{array}$ & Qualitativo & $\begin{array}{l}\text { Argentina } \\
\text { Medicina } \\
\text { Infantil }\end{array}$ \\
\hline 6. & $\begin{array}{l}\text { Jucá, V.D.S; Vorcaro, } \\
\text { A. M. R. / } 2018\end{array}$ & $\begin{array}{l}\text { Adolescência em atos e } \\
\text { adolescentes em ato na } \\
\text { clínica psicanalítica }\end{array}$ & $\begin{array}{l}\text { Estudo } \\
\text { Reflexivo }\end{array}$ & $\begin{array}{l}\text { Brasil/ Psicol. } \\
\text { USP }\end{array}$ \\
\hline 7. & $\begin{array}{l}\text { Fonseca, P. H. N; } \\
\text { Silva, A. C; Botti, N. } \\
\text { C. L. / } 2018\end{array}$ & $\begin{array}{l}\text { Autolesão sem intenção } \\
\text { suicida } \\
\text { adolescentes }\end{array}$ & $\begin{array}{l}\text { Quantitativo } \\
\text { Exploratório }\end{array}$ & $\begin{array}{l}\text { Brasil / Arq. bras. } \\
\text { Psicologia RJ. }\end{array}$ \\
\hline 8. & $\begin{array}{l}\text { Otto, S. Cr; Santos, K. } \\
\text { A. D. / } 2016\end{array}$ & $\begin{array}{l}\text { O Tumblr e sua relação } \\
\text { com práticas } \\
\text { autodestrutivas: o caráter } \\
\text { epidemico da autolesão }\end{array}$ & $\begin{array}{l}\text { Análise } \\
\text { Discursiva }\end{array}$ & $\begin{array}{l}\text { Brasil } \\
\text { Psicologia } \\
\text { Revista }\end{array}$ \\
\hline 9. & Castro, C, J. / 2014 & $\begin{array}{l}\text { Autolesión no suicida en } \\
\text { adolescentes peruanas: } \\
\text { Una aproximación } \\
\text { diagnóstica y } \\
\text { psicopatológica. }\end{array}$ & $\begin{array}{l}\text { Qualitativo } \\
\text { Exploratório }\end{array}$ & $\begin{array}{ll}\text { Peru / } & \text { Revista } \\
\text { de } & \text { Neuro- } \\
\text { Psiquiatría }\end{array}$ \\
\hline 10. & $\begin{array}{l}\text { Cortés, A, A. et al., / } \\
2011\end{array}$ & $\begin{array}{l}\text { Factores de riesgo } \\
\text { asociados con el intento } \\
\text { suicida y criterios sobre } \\
\text { lo ocurrido } \\
\text { adolescentes. }\end{array}$ & $\begin{array}{l}\text { Analítico } \\
\text { Caso-controle }\end{array}$ & $\begin{array}{l}\text { Cuba / Revista } \\
\text { Cubana de } \\
\text { Medicina } \\
\text { General Integral }\end{array}$ \\
\hline
\end{tabular}




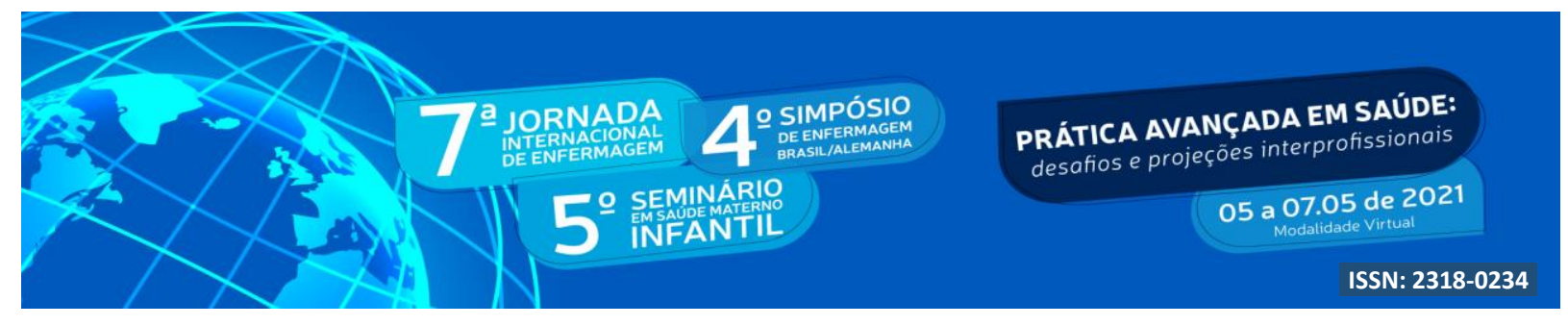

\begin{tabular}{|l|l|lr|l|l|}
\hline 11. & Drieu, D; Proia- & Ataques ao corpo & e & Analítico & França / Ágora: \\
& Lelouey, N; Zanello, \\
F. /2011 & & na & Reflexivo & Estudos em \\
adolescência & & & & Teoria \\
& & & & & Psicanalítica \\
\hline
\end{tabular}

Fonte: dados da pesquisa, 2020; Elaborada pelas autoras.

No que se refere à procedência geográfica, 07 estudos foram desenvolvidos na América do Sul, sendo que o Brasil apresentou 04 artigos ${ }^{(4,6,7,8)}$, seguido da Argentina com dois artigos ${ }^{(3,5)}$ e posteriormente Peru, com um artigo ${ }^{(9)}$. Na América Central, evidenciou-se um estudo Cubano ${ }^{(10)}$. Já, no continente europeu há três estudos, realizados em Portugal ${ }^{(1)}$, Espanha $^{(2)}$ e França ${ }^{(11)}$, com um artigo cada.

Em relação ao delineamento metodológico, predominou a abordagem qualitativa presente em quatro estudos ${ }^{(3,4,5,9)}$, sendo um caracterizado por ser do tipo exploratório ${ }^{(9)} \mathrm{e}$ um ${ }^{(3)}$ transversal. Evidenciou-se, ainda, um quatitativo exploratório (7), um misto (1) e um estudo de caso ${ }^{(2)}$. Além disso, apresentaram-se estudos do tipo: reflexivo (6), analítico casocontrole $^{(10)}$, analítico reflexivo ${ }^{(11)}$ e análise discursiva ${ }^{(8)}$, com um artigo cada.

Os estudos evidenciaram que o comportamento autolesivo tem se apresentado como um problema sanitário de crescente relevância, com prevalência significativa entre adolescentes. Entre os fatores associados a este comportamento apresentou-se o relacionamento conflituoso com os pais, o sentimento de desesperança, a vivência de violência doméstica e o desejo de sentir dor com intuito de aliviar emoções, experienciados pelos adolescentes (GIUSTI, 2013). Ademais, percebeu-se na literatura a alta incidência de adolescentes do sexo feminino, apresentando-se como as principais protagonizadoras deste fenômeno (KLONSKY et al., 2015).

A prática deste comportamento sem ideação suicida apresenta-se principalmente na forma de cortes nos braços e pernas. Percebe-se a prevalência das autolesões justificadas pela necessidade de produzir alívio momentâneo, buscar autoafirmação e atenuar sensações negativas como tristeza (GIUSTI, 2013). Neste viés, evidencia-se que a maioria dos adolescentes que desenvolvem o comportamento autolesivo após as práticas não possui sentimento de arrependimento, além de não apresentarem crítica sobre tais situações. Dessa 


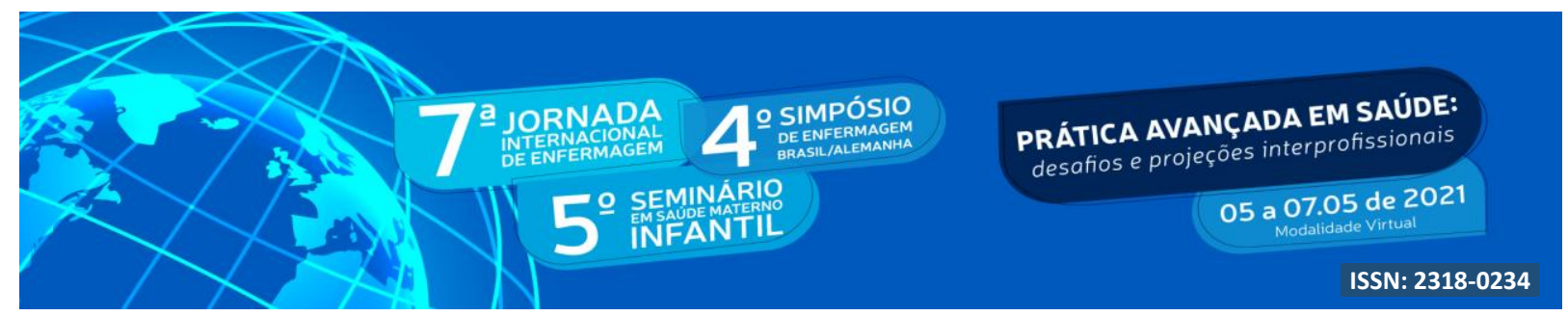

forma, favorecendo que o mesmo se estenda até a vida adulta (CARDOSO, 2015).

Entre as principais formas de tratamento ofertadas aos adolescentes que praticam autolesões, tem-se principalmente a abordagem do grupo familiar. Percebe-se, a necessidade de intervenções nos ambientes de saúde e escola através de estratégias abrangentes que possam identificar fatores e comportamentos de risco, além de dar subsídio e apoio social aos pais ou responsáveis (CARDOSO, 2015). Evidencia-se, que tal abordagem, apresenta-se relevante quando realizada por profissionais capacitados para a mesma. Os profissionais precisam buscar compreensão dos eventos e informações acerca dos mesmos que acrescentem no cuidado e possam prevenir novas incidências (LOPES, 2018).

\section{CONCLUSÃO}

Conclui-se que as evidências científicas relacionadas ao comportamento autolesivo entre adolescentes apontam principalmente as causas $e$ fatores envolvidos no desenvolvimento desse comportamento, tendo as adolescentes do sexo feminino como as principais protagonistas destes eventos. Nesse sentido, entre as principais causas para este comportamento, destacam-se problemas familiares, sentimentos de tristeza, vivência de violência doméstica e necessidade de atenuar sensações negativas. Por fim, percebe-se que o comportamento autolesivo, apresenta-se como um problema sanitário de saúde relevante na atualidade e que necessita implementação de estratégias abrangentes no setor familiar e escolar para que possam ser desenvolvidas estratégias que previnam tais incidências no público adolescente, evitando que o mesmo perpetue até a vida adulta.

\section{REFERÊNCIAS}

CARDOSO, B. C.C. A escarificação na adolescência: A problemática do Eu-Pele a partir do método de Rorschach. Mestrado em PSICOLOGIA CLÍNICA E CULTURA Instituição de Ensino: UNIVERSIDADE DE BRASÍLIA, Brasília Biblioteca Depositária: Biblioteca Central / 2015.

CUMMINGS, J.D. Self-injury: The Ultimate Teen Guide. Rowman \& Littlefield, 2015. FONSECA, P.H N.D.A, et al. Autolesão sem intenção suicida entre adolescentes. Arquivos 


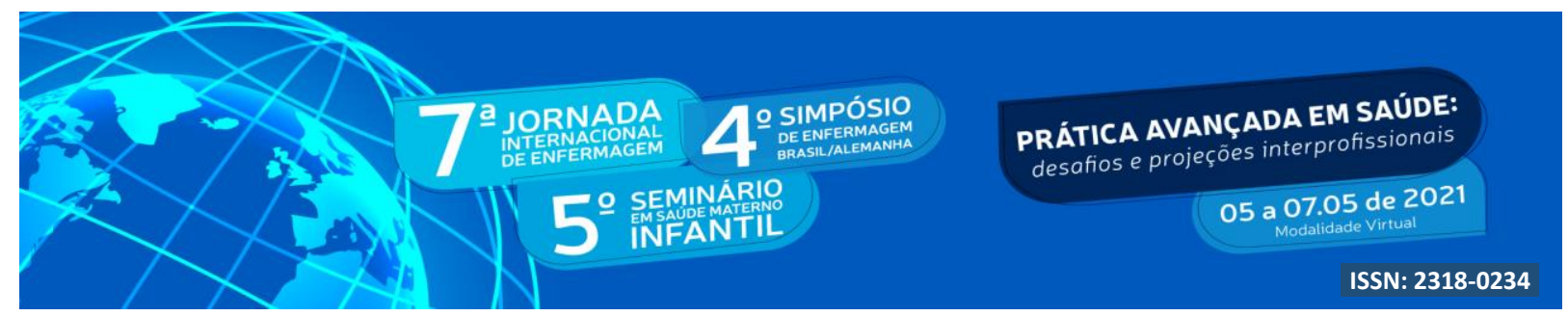

p. 246-258. Disponível em: <http://pepsic.bvsalud.org/pdf/arbp/v70n3/17.pdf> Acesso em: 15 out. 2019.

GIUSTI, J.S. Automutilação: características clínicas e comparação com pacientes com transtorno obsessivo-compulsivo. Doutorado em PSIQUIATRIA Instituição de Ensino: UNIVERSIDADE DE SÃ̃ PAULO, São Paulo Biblioteca Depositária: Biblioteca Central da FMUSP / 2013.

KLONSKY, E. D. et al. The functions of nonsuicidal self-injury: converging evidence for a two-factor structure. Child and adolescent psychiatry and mental health, v. 9, n. 1, p. 44, 2015.

LOPES, N. M. O "JOGO BALEIA AZUL": REPRESENTAÇÕES SOCIAIS DO SUICÍDIO JUVENIL NA MÍDIA MINEIRA ON LINE. Mestrado em ECONOMIA DOMÉSTICA Instituição de Ensino: UNIVERSIDADE FEDERAL DE VIÇOSA, Viçosa Biblioteca Depositária: Biblioteca Central da UFV e Setorial do Departamento de Economia Doméstica / 2018.

MENDES, K.D.S.; SILVEIRA, R.C.C.P. \& GALVÃO, C.M. (2008). Revisão integrativa: método de pesquisa para incorporação de evidências na saúde e na enfermagem. Texto \& Contexto Enfermagem, 17(4), 758-764.

RAUPP, C. S; MARIN, A. H; MOSMANN, C.P. Comportamentos autolesivos e administração das emoções em adolescentes do sexo feminino. Psicologia Clínica, 2018, vol. 30, no 2, p. 289-308.

SANTOS, L. C. S.; FARO, A. Aspectos conceituais da conduta autolesiva: Uma revisão teórica. Psicologia em Pesquisa, 2018, vol. 12, no 1, p. 5-14.

SILVA, M.F.D. A.; SIQUEIRA, A. C. O perfil de adolescentes com comportamentos de autolesão identificados nas escolas estaduais em Rolim de Moura-RO. Revista Farol, 2017, vol. 3, no 3, p. 5-20.

SOUSA, L.L.M. et al. (2017). Metodologia de Revisão Integrativa da Literatura em Enfermagem. Revista Investigação em Enfermagem, 2, 17-26.

WHO. World Health Organization Preventing suicide: a global imperative. Geneva; 2014. 